\title{
Atom Dynamics at the Gas-Catalysts Interface with Atomic Resolution
}

\author{
C. Kisielowski ${ }^{1,2}$, S. Helveg ${ }^{3}$, G. Yuan ${ }^{4}$, H. Frei ${ }^{4}$, \\ 1. Joint Center for Artificial Photosynthesis (JCAP), Lawrence Berkeley National Laboratory, 1 \\ Cyclotron Road, Berkeley, CA 94720 (USA). \\ 2. National Center for Electron Microscopy, Lawrence Berkeley National Laboratory, 1 Cyclotron \\ Road, Berkeley, CA 94720 (USA). \\ 3. Haldor Topsøe A/S, Nymøllevej 55, DK-2800 Kgs. Lyngby, Denmark. \\ 4. Physical Biosciences Division, Lawrence Berkeley National Laboratory, 1 Cyclotron Road, \\ Berkeley, CA 94720 (USA)
}

Transmission electron microscopy at the edge to technological capabilities enables the detection of single atoms and their dynamic behavior on a length scale of pico meters in real time. It is now understood that a previously unexplained atom dynamic observable during recordings at high resolution is intimately linked to the relaxation and thermalization of electron beam-induced sample excitation [1]. Resulting contrast fluctuations are beam current dependent and largely contribute to a contrast mismatch between experiments and theory if not considered. If explored, they open the possibility to maintain pristine object structures of catalysts as shown in Figure 1 and to study functional behavior of nanocrystals and single molecules at the atomic level in real time [1].

In the past in-situ transmission electron microscopy at elevated temperature and pressure (sometimes referred to as "E-TEM") often lacks the needed signal-to-noise rations to detect single atoms or molecules and their dynamics on catalytic surfaces. An in-situ instrument operated at H. Topsoe A/S, Denmark, is used to implement in-line holography at variable dose rates and voltages [2] to show that the approach drastically minimizes the effects of beam-sample interactions and boost signal-to-noise ratios [3].

Examples are given in Figure 2. It shows $\mathrm{Co}_{3} \mathrm{O}_{4}$ catalysts that are observed during exposure to a reducing or oxidizing environment at room temperature and a finite partial pressure. While the acquisition of single images with high dose rates typically yields pictures that do not reveal detailed surface structures even in vacuum (Figure 2a, inset), the presence of surface reconstructions formed by only few atoms can be detected in the phase image of low dose-rate in-line hologram ( arrow in Fig. 2a), $\left.1.710^{-6} \mathrm{mbar}\right)$. An exposure of such surfaces to a reducing $\mathrm{CO}$ atmosphere causes surface roughening on a scale of 1-3 atomic layers (Figure $2 \mathrm{~b}$ ), $\mathrm{p}(\mathrm{CO})=1.410^{-2} \mathrm{mbar}$ ). In contrast, an exposure of the previously reduced $\mathrm{Co}_{3} \mathrm{O}_{4}$ surfaces to water vapor in the range $1.410^{-2} \mathrm{mbar} \leq \mathrm{p}\left(\mathrm{H}_{2} \mathrm{O}\right) \leq 1.410^{-2} \mathrm{mbar}$ stimulates molecule attachments (Figure $2 \mathrm{c}$ ). A continuous amorphous monolayer eventually forms and covers all surfaces (Figure $2 \mathrm{~d}$ ). Contributions from the imaging electron beam $(80 \mathrm{kV})$ are present and are currently evaluated. Thus, our approach expands the applicability of in-situ transmission electron microscopy to include observations at the single atom/molecule level. It allows to better support chemical sciences and helps building a foundation that will ultimately enable atomic scale observations of functional behavior in chemically meaningful environments.

References:

[1] C. Kisielowski et al., Phys. Rev. B 88 (2013) 024305.

[2] B. Barton et al. Microsc.Microanal. 18 (2012) 1606-1607.

[3] S. Helweg et al. Sub. Micron, 2014 
[4] This work is performed by the Joint Center for Artificial Photosynthesis, a DOE Energy Innovation Hub, supported through the Office of Science of the U.S. Department of Energy under Award Number DE-SC000499. Operation of the TEAM microscope at NCEM is supported by the Office of Science, Office of Basic Energy Sciences of the U.S. Department of Energy under Contract No. DE-AC02-05CH11231.

a) Low dose rate conditions: $69 \mathrm{e} / \mathrm{A} / \mathrm{s}$
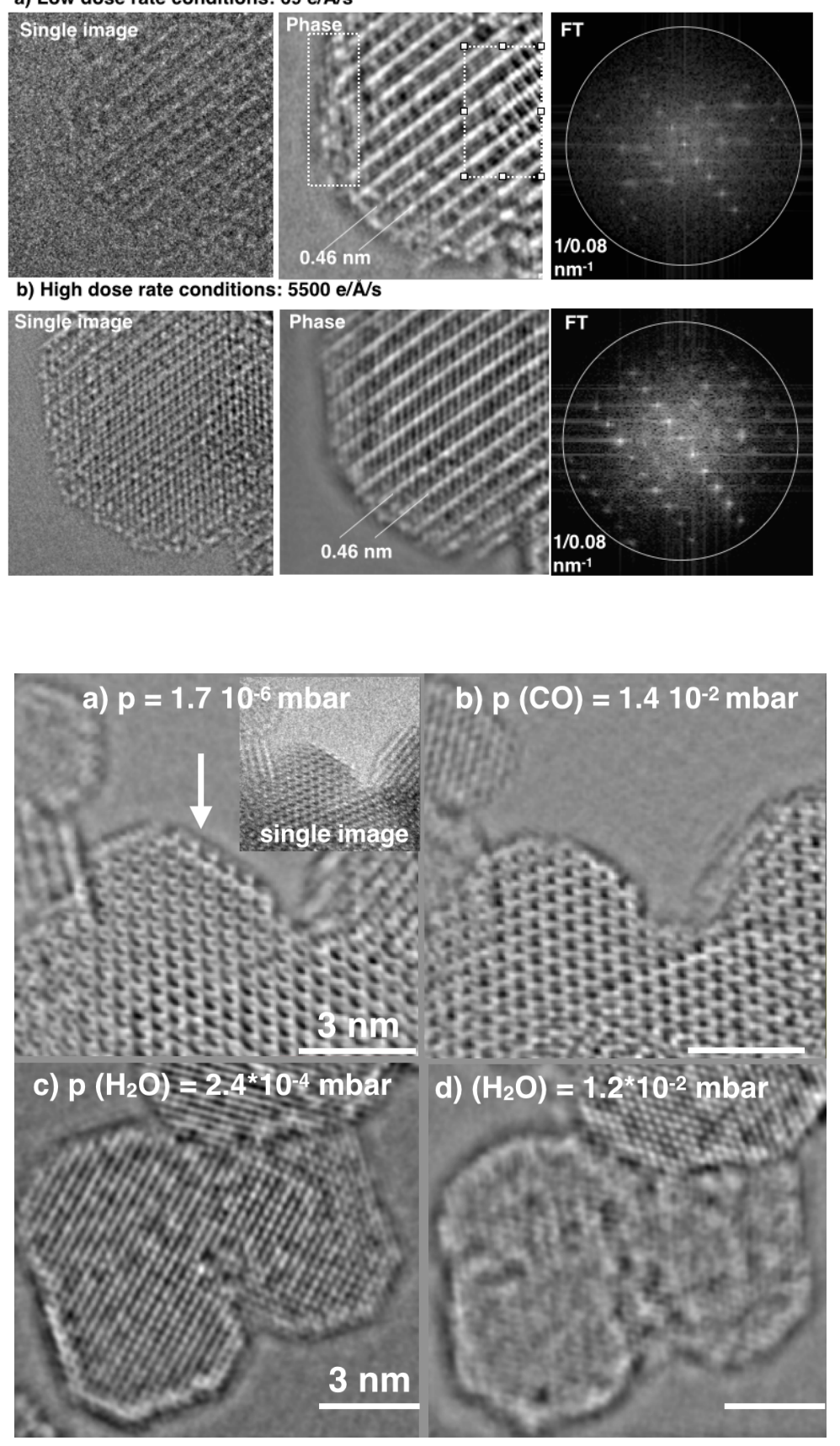

Figure 1. Single images, phase images of electron exit waves reconstructed from a focus series of 60 images, and the Fourier Transform of the exit wave function (= nano diffraction pattern) are shown for successive recordings first using a low dose rate (a) and then a high dose rate (b). Beam-induced surface alterations are revealed comparing the phase images (boxes). Only, images recorded in low dose rate conditions depict the pristine catalyst structure from the sample in the base vacuum of the TEAM0.5 $(80 \mathrm{kV})$.

Figure 2. Phase images of a $\mathrm{Co}_{3} \mathrm{O}_{4}$ catalysts exposed to different environments during in situ observation. a) A surface reconstruction is seen at the vacuum / catalyst interface (arrow); a single image is inserted where the $\mathrm{S} / \mathrm{N}$ ratio does not suffice to see the reconstruction. b) Surface roughening of the particle shown in a) by an exposure to a reducing $\mathrm{CO}$ gas. c, d) Exposure to an increasing pressure of water vapor. A monolayer of molecules that covers the surfaces grows that is visible in a plane-view configuration because of the improved signal to noise ratio of the reconstructed phase image. The dose rate is $280 \mathrm{e} / \AA^{2}$ s. [3] 